\title{
Proceeding
}

8th INSHS International Christmas Sport Scientific Conference, 5-7 December 2013. International Network of Sport and Health

Science. Szombathely, Hungary

\section{Physical activity benefits for Alzheimer's disease patients (A Review)}

\author{
Genti Pano
}

Sports University of Tirana, Sports Sciences Research Institute, Tirana, Albania.

\begin{abstract}
Pano, G. (2014). Physical activity benefits for Alzheimer's disease patients. (A Review). J. Hum. Sport Exerc., 9(Proc1), pp.S319-S325. Alzheimer's disease (AD) is a chronic and degenerative disease which is the main cause for dementia in older adults. It is well known that exercise can reduce the risk level for vascular risk factors, heart diseases (Blair et al., 1996), atherosclerosis (Lakka et al., 2001), stroke (Kurl et al., 2001) and diabetes (Seals et al., 1984; Houmard et al., 1996), diseases that can increase the risk for dementia and $A D$ (Gustafson et al., 2003). Main objective of this study was to review the latest literature recomendations regarding the most appropriate exercise testing, programming and types of physical activity that Alzheimer's disease patients should practice. The search has been made mainly, focusing in PubMed/MEDLINE, for randomized controlled trial studies which used PA intervention as a weapon for delaying or treating symtoms of $A D$ patients. There are clear evidences which shows that AD patients can benefit from all types of occupational PA and have a positive effect on their psycho-social and cognitive functioning. $A D$ patients who engage in $P A$, also have positive effect in motor abilities, activities of daily life and makes them more independent from others. Further studies for $A D$ patients involving a larger number of subjects and different types of individualised exercise interventional programs are necessary to be conducted. Key words: ALZHEIMER DISEASE, PHYSICAL ACTIVITY, EXERCISE THERAPY.
\end{abstract}

Corresponding author. Sports University of Tirana, Sports Sciences Research Institute, Tirana, Albania.

E-mail: gentip2005@hotmail.com

8th INSHS International Christmas Sport Scientific Conference, 5-7 December 2013. International Network of Sport and Health Science. Szombathely, Hungary.

JOURNAL OF HUMAN SPORT \& EXERCISE ISSN 1988-5202

(c) Faculty of Education. University of Alicante

doi:10.14198/jhse.2014.9.Proc1.15

VOLUME 9 | Proc1 | 2014 | S319 


\section{INTRODUCTION}

\section{Alzheimer's Disease}

Alzheimer's disease (AD) it's a chronic and degenerative disease which is the main cause for dementia in older adults. The disease was first recognized in 1907 by the German psychiatrist Alois Alzheimer. The prevalence of Alzheimer's disease rises exponentially with age. After age 65, the percentage of affected people approximately doubles with every decade of life. The highest rate of Alzheimer's disease occurs in people age 85 and over. Alzheimer's disease is more common in women than in men. Aging brings an increased incidence of cognitive and physical decline, especially in those in the eighth decade and beyond (NIA, 2007) Evidences indicates that aging is accompanied by a systematic decline in performance of a wide variety of cognitive tasks, observed both in the laboratory setting and in everyday life (Dixon et al., 2004). Several studies have suggested that differential susceptibility to age-related cognitive decline or to Alzheimer's disease is related to variables such as occupation (Schooler et al., 1999), professional or leisure activities (Wilson et al., 2002), and lifestyle (Fillit et al., 2002). A recent Mayo Clinic special report (Mayo Clinic Health information) on how exercise helps Alzheimer's patient's stated specific benefits, in addition to the general ones of improved strength, endurance, cardiovascular health, blood pressure control, and reduced risk of falling. At the present time, there is no cure for this disease and, for the most part, treatment has been limited.

Some of Alzheimer's disease symptoms include:

- Deterioration of language and perception,

- Judgment problems that compromise the person's ability to carry out activities of daily living, and

- Behavioral problems such as agitation and paranoia

- Although the life expectancy among persons with $A D$ is diverse, early mortality is seen among persons who develop it early in life and in men

- However, persons with $A D$ can often live far years with this condition, dying eventually from pneumonia or other diseases

- The duration of Alzheimer's disease from time of diagnosis to death can be 20 years or more, with the average length 4 to 8 years

Exercise appears to stimulate neurogenesis (Van Praag et al., 1999), enhance neuronal survival (Barde, 1994), increase resistance to brain insults (Stummer et al., 1994; Carro et al., 2001) and increase synaptic plasticity (Lu \& Chow, 1999).

\section{Objectives}

- Main objective of this study was to review the literature of the last five years, regarding the most appropriate exercise testing, programming and type of physical activity that Alzheimer's disease patients should be involved.

- The search has been made mainly, focusing in PubMed/MEDLINE, for randomized controlled trial studies which used PA intervention as a weapon for delaying or treating symptoms of AD patients.

Selecting criteria:

- $\quad$ Only randomized controlled trials studies.

- $\quad$ Subjects over 55 years old

- $\quad$ Subjects diagnosed with AD (Males \& Females)

- Only publications in English language. 
- $\quad$ Subjects that used in the same time other medication treatments for physical and/or psychological problems.

\section{RESULTS}

Description of the studies:

From 71 possible papers, only 5 were identified as appropriate to be enrolled in this review.

Enrolled studies were:

1. Sharon M.A., 2003;

2. Williams C. L., et al 2008;

3. Cyarto E.V et al., 2010;

4. Baker L.D et al., 2010

5. Vidoni E.D et al., 2012.

Therapeutic regimens were from 2 weeks to 26 months and in all the studies exercise therapy was combined with other adjuvant therapies. All the Studies had a total of over 350 participants.

Subject's age was from 55 years old to 91 years old, also the main used exercise therapies were:

- Exercise therapy; aerobic exercises/150 min/week, walking on treadmill,

- Exercise in home conditions 150 min/week, moderate intensity

- $\quad$ Exercise sessions, flexibility, balance, aerobics and resistance training with weights.

In Sharon MA, 2003 study, 24 individuals, aged 54 to 88 were recruited. They participated from for two to eight semesters in program consisted in 16 to 20 exercise sessions and 10 community activity sessions per semester. Half of the weekly exercise sessions included memory and language stimulation activities. Students, supplemented by family caregivers, supervised the sessions. Exercise sessions consisted of flexibility, balance, aerobic, and weight resistance activities. Pre-participation and semiannual post-testing of aerobic fitness and duration and upper and lower body strength was done. Highly significant fitness gains $(p<.001)$ were achieved in the six-minute walk test, upper and lower body strength, and duration of aerobic exercise. Five participants aged 86 to 91 , completed six to eight semesters and were doing 27 to 45 minutes of aerobics per session at program's end. Five participants scored within the normal range for age-matched healthy active adults on the six minute walk test at baseline. Nine others achieved and three exceeded the normal range during treatment. 
Table 1. Interventions characteristics.

\begin{tabular}{|c|c|c|c|c|}
\hline Study ID & $\begin{array}{l}\text { Number of } \\
\text { the subjects }\end{array}$ & $\begin{array}{l}\text { Age in } \\
\text { years }\end{array}$ & Duration & Type of intervention \\
\hline Sharon M.A., 2003 & 29 & $54-91$ & 2-8 Weeks & $\begin{array}{l}\text { Exercise sessions, flexibility, } \\
\text { balance, aerobic exercises, resistance } \\
\text { exercises, and exercises with weights }\end{array}$ \\
\hline Williams C. L., et al 2008 & 45 & 60 & 16 Weeks & $\begin{array}{l}5 \text { times/week individual sessions, } \\
\text { controlled walking and social } \\
\text { conversations }\end{array}$ \\
\hline Cyarto E.V et al., 2010 & 230 & $54-88$ & 24 Weeks & $\begin{array}{l}\text { Exercise program in home } 150 \\
\mathrm{~min} / \text { week, moderate PA }\end{array}$ \\
\hline Baker L.D et al., 2010 & 28 & $57-83$ & 6 Months & Aerobic exercises and stretching \\
\hline Vidoni E.D et al., 2012 & $\begin{array}{l}\text { Non } \\
\text { specified }\end{array}$ & $55-$ & 26 Months & $\begin{array}{l}\text { Aerobic exercises } 150 \mathrm{~min} / \text { week } \\
\text { walking on treadmill }\end{array}$ \\
\hline
\end{tabular}

Cognitive decline was slowed and mood improved. Williams et al., 2008 study, was a three-group, repeated-measures design with random assignment to treatment group. Forty-five nursing home residents with moderate to severe $A D$ were randomly assigned to a 16-week program of comprehensive exercise, supervised walking or social conversation. The purpose of this study was to compare the effects of 16 weeks of a comprehensive exercise routine to supervised walking and social conversation on depression in nursing home residents with Alzheimer's disease (AD). Major outcome variables were depression measured by the Cornell Scale for Depression in Dementia, mood measured by the Dementia Mood Assessment Scale and the Alzheimer's Mood Scale, and affect measured by the Observed Affect Scale. Results have shown that depression was reduced in all three groups with some evidence of superior benefit from exercise. Cyarto et al., (2010) study 230 community-dwelling participants diagnosed with Alzheimer's disease were recruited. Participants were randomly separated into two treatment groups: usual care group or 24-week home based program consisting of 150 minutes per week of tailored moderate physical activity. The primary outcome measure of this study was cognitive decline as measured by the change from baseline in the total score on the Alzheimer's disease Assessment Scale-Cognitive section. Secondary outcomes of interest had included behavioural and psychological symptoms, quality of life, functional level, career burden and physical function (strength, balance, endurance, physical activity). Primary endpoints were measured at six and twelve months following the baseline assessment. Baker et al., 2010 study was a randomized controlled design, were twenty-eight adults (57-83 y old) meeting 2-h tolerance test criteria for glucose intolerance completed 6 months of aerobic exercise or stretching, which served as the control. The primary cognitive outcomes of this paper included measures of executive function (Trails B, Task Switching, Stroop, Self-ordered Pointing Test, and Verbal Fluency). Other outcomes included were; memory performance (Story Recall, List Learning), measures of cardiorespiratory fitness (obtained via maximal-graded exercise treadmill test), glucose disposal during hyperinsulinemic-euglycemic clamp, body fat, and fasting plasma levels of insulin, cortisol, brain-derived neurotrophic factor, insulin-like growth factor-1, amyloid- $\beta$ (A $\beta 40$ and A $\beta 42)$. Vidoni et al., (2012) paper porpoise was to report the protocol of a current, community-based pilot study of aerobic exercise for $A D$ to 
guide future investigation. This manuscript provides 1) an overview of possible benefits of exercise in those with dementia, 2) a rationale and recommendations for implementation of a community-based approach, 3) recommendation for implementation of similar study protocols, and 4) unique challenges in conducting an exercise trial in AD.

\section{DISCUSSION}

It remains uncertain how exercise may benefit brain health and cognitive function in $A D$, now one of our most costly and pervasive age-related diseases. Aerobic exercise may have its greatest remediating effect on frontal brain regions that are most vulnerable to aging, and for glucose intolerant adults with an increased risk of cognitive decline and $A D$, to deleterious consequences of diabetes- and AD-related vascular dysfunction (Manschot et al., 2006; Jellinger, 2002; Stopa et al., 2008). In Williams et al., 2008 study, exercise approaches to treatment of depression in nursing home residents with severe $A D$ showed a clear benefit of the participants. The results of this study were limited due to the small sample size. There was evident benefit of exercise over the attention-control intervention (social conversation) on some of the mood outcomes. In this study the authors stated that, chair-based exercise in which non-ambulatory individuals could participate should be investigated and that social conversation, originally believed to be an attention-control, evidently had some beneficial effect although it may be necessary to provide much more of it than exercise to gain that effect. In Cyarto et al., 2010, concluded that the findings have the potential to inform practitioners about successful strategies and provide the impetus for translation into community programs. This study focused the participant's cognitive, clinical symptoms, functional level and QoL but also on the indirect positive effects that may be experienced by the caregiver (Cyarto et al., 2010). In Baker et al., 2010, results showed that, six months of aerobic exercise improved cognitive performance on tasks of executive function including selective and divided attention, cognitive flexibility, and working memory in older adults with glucose intolerance. In addition, circulating levels of the AD biomarker A $\beta 42$ tended to decrease for subjects in the aerobic group relative to controls. Also in this study (Baker et al., 2010), executive function and insulin sensitivity of the enrolled subjects were improved with aerobic exercise, finding that implicates a potential benefit of improved glucose metabolism on cognitive processes. The results of this study suggest that aerobic exercise may also have an impact on circulating $A \beta 42$ given that plasma levels tended to decrease for subjects in the aerobic exercise group relative to controls. They also reported a similar change in A $\beta 42$ levels in response to a 6-month trial of aerobic exercise for older adults with mild cognitive impairment. Also they have stated that exercise-induced improvements in insulin sensitivity, cerebral blood flow, and other metabolic parameters may contribute to the observed cognitive benefits and that increased physical activity level is a potent non-pharmacological intervention for physiological symptoms associated with impaired glucose metabolism and T2DM, conditions that confer increased risk of $A D$.

\section{CONCLUSIONS \& RECOMMENDATIONS}

Based on the reviewed studies we can say that:

- Sharon M., 2003 study, has clearly proved that older individuals with dementia can safely participate in an ongoing vigorous exercise program and achieve significant physical fitness and mood gains. Furthermore, it demonstrated that college students are an available, safe, reliable, and affordable source of transportation, one-to-one exercise supervision, and companionship for elderly persons with dementia.

- Exercise therapy is one of the best ways for treating $A D$ patients and results show that exercise benefits are equal to medications. 
- In future studies of exercise, inclusion of a 'usual care' control group must be carefully considered.

- The results of Baker L.D et al., 2010 suggest that 6 months of moderate to high intensity aerobic exercise may influence circulating levels of $A \beta 42$, a finding with potential implications for $A D$ pathology.

- Since AD patients are unlikely to start an exercise program, it is necessary that all the caregivers must be trained in order to encourage AD patients to exercise.

- More research is needed to clarify the relative benefits of different types of exercise in conjunction with or without pharmacological intervention.

\section{REFERENCES}

1. Arkin, S.M. (2003). Student-led exercise sessions yield significant fitness gains for Alzheimer's patients. American Journal of Alzheimer 's disease and Other Dementias, 18(3), pp.159.

2. Barde, Y.A. (1994). Neurotrophins: a family of proteins supporting the survival of neurons. Prog Clin Biol Res, 390, pp.45-56.

3. Baker, L.D., Frank, L.L., Foster-Schubert, K., Green, P.S., Wilkinson, C.W., McTiernan, A., Brenna, A., Cholerton,B.A., Plymate, S.R., Fishel, M.A., Watson, G. S., Duncang, G.E., Mehta, P.D. \& Craft, S. (2010). Aerobic Exercise Improves Cognition for Older Adults with Glucose Intolerance, A Risk Factor for Alzheimer's Disease. Published in final edited form as: J Alzheimers Dis, 22(2), pp.569579.

4. Carro, E., Trejo, J.L., Busiguina, S. \& Torres-Aleman, I. (2001). Circulating insulin-like growth factor I mediates the protective effects of physical exercise against brain insults of different etiology and anatomy. J Neurosci, 21, pp.5678-84.

5. Cyarto E.V., Cox, K.L., Almeida, P.O., Flicker, L., Ames, D., Byrne, G., Hill, K.D., Beer, C.D., Logiudice, D., Appadurai, K., Irish, M., Renehan, E. \& Lautenschlager, N.T. (2010). The fitness for the Ageing Brain Study II (FABS II): protocol for a randomized controlled clinical trial evaluating the effect of physical activity on cognitive function in patients with Alzheimer's disease. Cyarto et al; licensee BioMed Central Ltd.

6. Dixon, R.A., Bäckman, L. \& Nilsson, L.G.R. (2004). New Frontiers in Cognitive Aging. Oxford, UK: Oxford University Press.

7. Fillit, H.M., Butler, R.N., O'Connell, A.W. et al. (2002). Achieving and maintaining cognitive vitality with aging. Mayo Clin Proc, 77, pp.681-696.

8. Jellinger, K.A. (2002). Alzheimer disease and cerebrovascular pathology: an update. J Neural Transm, 109, pp.813-836.

9. Lu, B. \& Chow, A. (1999). Neurotrophins and hippocampal synaptic transmission and plasticity. J Neurosci Res, 58, pp.76-87.

10. Manschot, S.M., Brands, A.M., Van Der Grond, J., Kessels, R.P., Algra, A., Kappelle, L.J. \& Biessels, G.J. (2006). Brain magnetic resonance imaging correlates of impaired cognition in patients with type 2 diabetes. Diabetes, 55, pp.1106-1113.

11. Mayo Clinic Health Information: Alzheimer's Disease: How exercise helps. Available on the following website: www.walgreens. com/library/lifestyle/fitness/special/spec5.jhtml.

12. National Institute On Aging. (2007). Growing older in america: the health and retirement study. In: Karp F, editor. Bethesda, MD: National Institutes of Health.

13. Schooler, C., Mulatu, M.S. \& Oates, G. (1999). The continuing effects of substantively complex work on the intellectual functioning of older workers. Psychol Aging, 14, pp.483-506. 
14. Stopa, E.G., Butala, P., Salloway, S., Johanson, C.E., Gonzalez, L., Tavares, R., Hovanesian, V., Hulette, C.M., Vitek, M.P. \& Cohen, R.A. (2008). Cerebral cortical arteriolar angiopathy, vascular beta-amyloid, smooth muscle actin, Braak stage, and APOE genotype. Stroke, 39, pp.814-821.

15. Stummer, W., Weber, K., Tranmer, B., Baethmann, A. \& Kempski, O. (1994). Reduced mortality and brain damage after locomotor activity in gerbil forebrain ischemia. Stroke, 25, pp.1862-9.

16. Van Praag, H., Christie, B.R., Sejnowski, T.J. \& Gage, F.H. (1999). Running enhances neurogenesis, learning, and long-term potentiation in mice. Proc Natl Acad Sci USA, 96, pp.1342731.

17. Vidoni E.D, Van Sciver, A., Johnson, D.K., Jinghua, H.E, Honea, R., Haines, R., Goodwin, J., Laubinger, M.P., Anderson, H.S., Kluding, P.M., Donnelly, F.J.E., Billinger, S.A., Burns, J.M., Volicer, L., Fabiszewski, K.J., Rheaume, Y.L. \& Lasch, K.E. (1988). Clinical management of Alzheimer's disease. Rockville, MD: Aspen. Wolf-Klein, G.P. 1993, New Alzheimer's drug expands your options in symptom management. Geriatrics, 48, pp.26-36.

18. Williams, C. L., Tappen, R.M. \& Lynn, C.E. (2008). Exercise training for depressed older adults with Alzheimer's Disease. NIH Public Access Author Manuscript. Aging Ment Health. Author manuscript; available in PMC 2008 July 21. Published in final edited form as: Aging Ment Health. 12(1), pp.72-80.

19. Wilson, R.S., Mendes De Leon, C.F., Barnes, L.L., et al. (2002). Participation in cognitively stimulating activities and risk of incident Alzheimer disease. JAMA, 287, pp.742-748 